\title{
Modified simultaneous integrated boost radiotherapy for large retroperitoneal malignant tumor: A case report
}

\author{
TAKUMA NOMIYA ${ }^{1}$, HIROKO AKAMATSU $^{2}$, MAYUMI HARADA $^{1}$, IBUKI OTA $^{2}$, \\ YASUHITO HAGIWARA ${ }^{2}$, MAYUMI ICHIKAWA ${ }^{2}$, MISAKO MIWA ${ }^{2}$, MASAOMI MIZUTANI ${ }^{3}$, \\ TOMOYUKI KATO ${ }^{4}$, AKIRA NAGAOKA ${ }^{4}$, YOSHIHIKO TOMITA ${ }^{4}$ and KENJI NEMOTO ${ }^{2}$ \\ ${ }^{1}$ National Institute of Radiological Sciences, Research Center Hospital for Charged Particle Therapy, Chiba 263-8555; \\ Departments of ${ }^{2}$ Radiation Oncology, ${ }^{3}$ Surgery and ${ }^{4}$ Urology, Yamagata University Hospital, Yamagata 990-9585, Japan
}

Received July 15, 2014; Accepted February 17, 2015

DOI: $10.3892 / 01.2015 .3095$

\begin{abstract}
The current study reports the case of a large retroperitoneal tumor treated with modified simultaneous integrated boost (SIB) radiotherapy. A 45-year-old female presented to the emergency department complaining of left abdominal pain and fever. A computed tomography scan detected a retroperitoneal tumor of $12 \times 16 \times 16 \mathrm{~cm}$, and a biopsy revealed a poorly-differentiated adenocarcinoma. The patient was diagnosed with a large adenocarcinoma originating from the left ureter, with no distant metastasis. Due to the patient's poor physical condition, surgery was not recommended, and the patient was referred to the Department of Radiation Oncology (Yamagata University Hospital, Yamagata, Japan). Modified SIB radiotherapy was administered following the acquisition of written consent from the patient. The total irradiation dose to the center of the tumor and to the surrounding healthy tissue was $\sim 96 \mathrm{~Gy} / 33$ fractions and $<60 \mathrm{~Gy} / 33$ fractions, respectively. At the end of the radiotherapeutic course, the tumor volume was reduced by $\geq 80 \%$, and the residual tumor was surgically resected. As a result of the resection, a complete pathological response was confirmed; the patient has been recurrence-free for $>3$ years with no complications. Modified SIB radiotherapy may be safely administered, with favorable outcomes. Complete recovery can be achieved with this technique, even in a patient with a large radioresistant tumor.
\end{abstract}

\section{Introduction}

Retroperitoneal tumors arise from the tissues of the retroperitoneal space, which includes the following structures: The adrenal glands, kidneys, ureter, aorta, inferior vena cava, pancreas

Correspondence to: Dr Takuma Nomiya, National Institute of Radiological Sciences, Research Center Hospital for Charged Particle Therapy, 4-9-1 Anagawa, Inage, Chiba 263-8555, Japan E-mail: t_nomiya@nirs.go.jp; t.nomiya@med.id.yamagata-u.ac.jp

Key words: retroperitoneal neoplasm, clinical trial, radiotherapy, technique (part), duodenum, colon (part), rectum, esophagus, lymph nodes, and soft tissue. Although in other sites the incidence of a benign tumor is higher than that of a malignant tumor, the incidence of a malignant tumor in the peritoneum is $\sim 4$ times higher than that of a benign tumor (1). The majority of retroperitoneal tumors are malignant soft tissue tumors, lymphoproliferative disorders, and malignancies arising from parenchymal tissues (1).

Approximately one-third of retroperitoneal tumors are soft tissue sarcomas, with liposarcoma and leiomyosarcoma accounting for $\sim 70 \%$ and $15 \%$ of reteroperitoneal sarcomas, respectively (2). The retroperitoneum is the second most common site of origin of soft tissue tumors (2), with $10-15 \%$ of soft tissue sarcomas arising from the retroperitoneum $(3,4)$. More rarely, Castleman's tumors (5), adult neuroblastoma (6), gastrinoma (7), solitary fibrous tumor of the pancreas (8), teratoma (9) and neurogenic tumors (10) have been reported to occur in this region. Due to the rarity of retroperitoneal large adenocarcinoma, literature regarding the surgical treatment of large retroperitoneal tumors is limited (11). Furthermore, no standard treatment exists for patients with inoperable large retroperitoneal tumors and thus, only palliative chemotherapy or radiotherapy or best supportive care is administered for these patients.

Retroperitoneal tumors show few clinical symptoms in the early phase, and are typically detected incidentally by computed tomography (CT), or by palpation when they become larger. The current study reports the case of a patient with a large retroperitoneal tumor, which was treated with definitive radiotherapy and surgery. Written informed consent was obtained from the patient.

\section{Case report}

A 45-year-old female presented to the emergency department of Yamagata University Hospital (Yamagata, Japan) complaining of left abdominal pain and fever. The patient was initially diagnosed with left hydronephrosis caused by retroperitoneal abscess. Ureteral catheterization was performed to treat the left hydronephrosis, and antibiotics (intravenous meropenem, $1 \mathrm{~g} /$ day) were prescribed. However, the abdominal pain and fever had not improved after 2 weeks, 

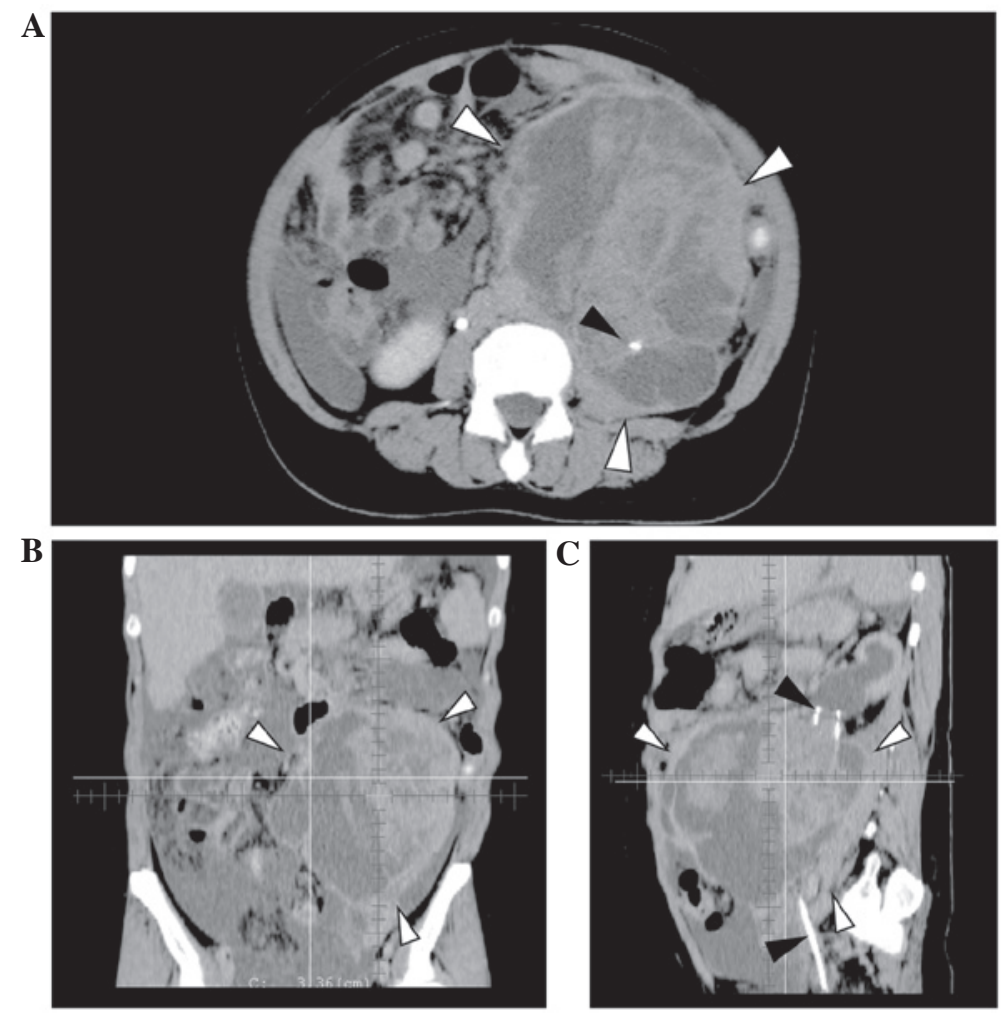

Figure 1. Computed tomography images prior to treatment. (A) Axial image showing the tumor of 12x16x16 cm located in the left abdomen (white arrowheads). The left ureter is involved in the tumor, and the ureteral catheter is visible in the tumor (black arrowhead). A small amount of ascites was also detected. (B) Coronal image showing the tumor with heterogeneous appearance and including cystic and solid components. (C) Sagittal image: Ureteral catheter penetrating the tumor (black arrowheads) and hydronephrosis were observed.
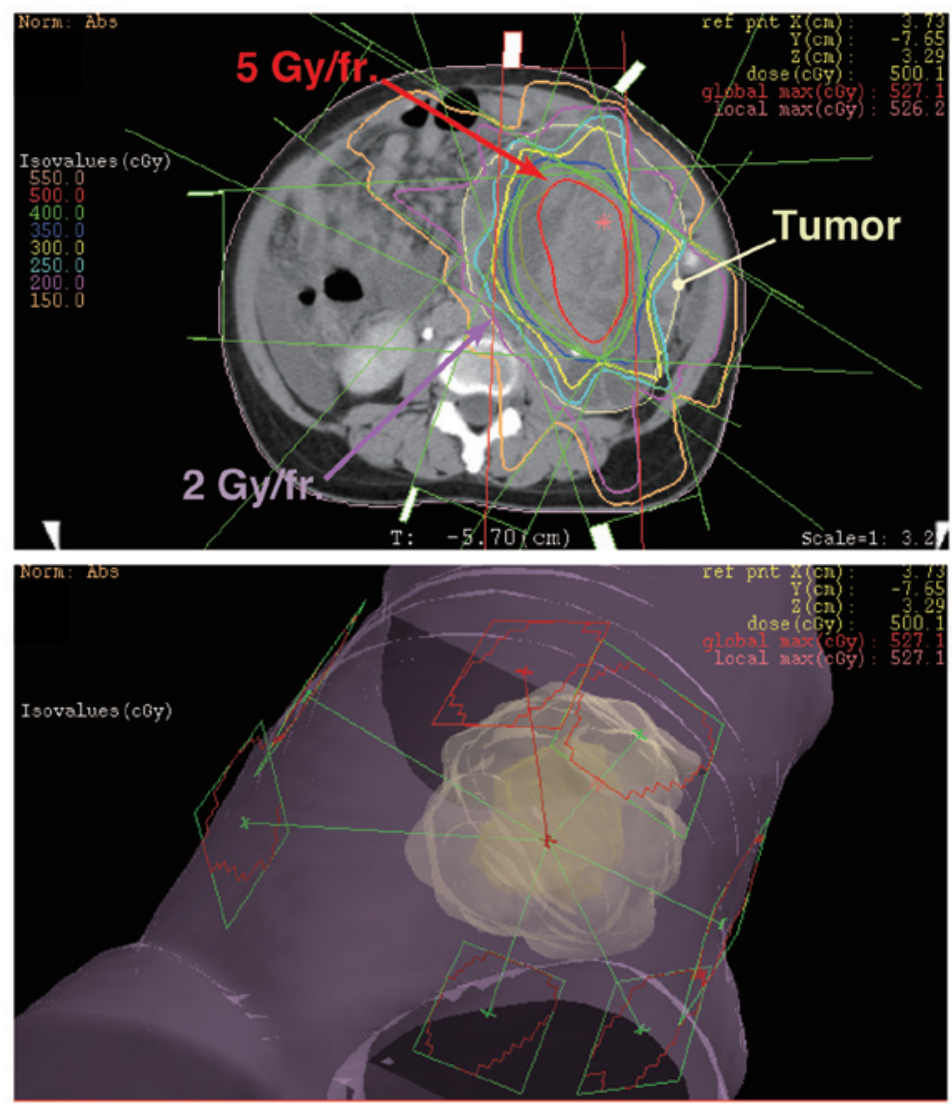

Figure 2. The initial radiotherapy planning with modified SIB technique. The center part of the tumor was irradiated with a single dose of $5 \mathrm{~Gy} /$ fraction, while the dose to the peripheral region including healthy tissues was suppressed to $<2 \mathrm{~Gy} /$ fraction. This treatment plan uses three-dimensional-conformal radiation therapy with eight static fields. 

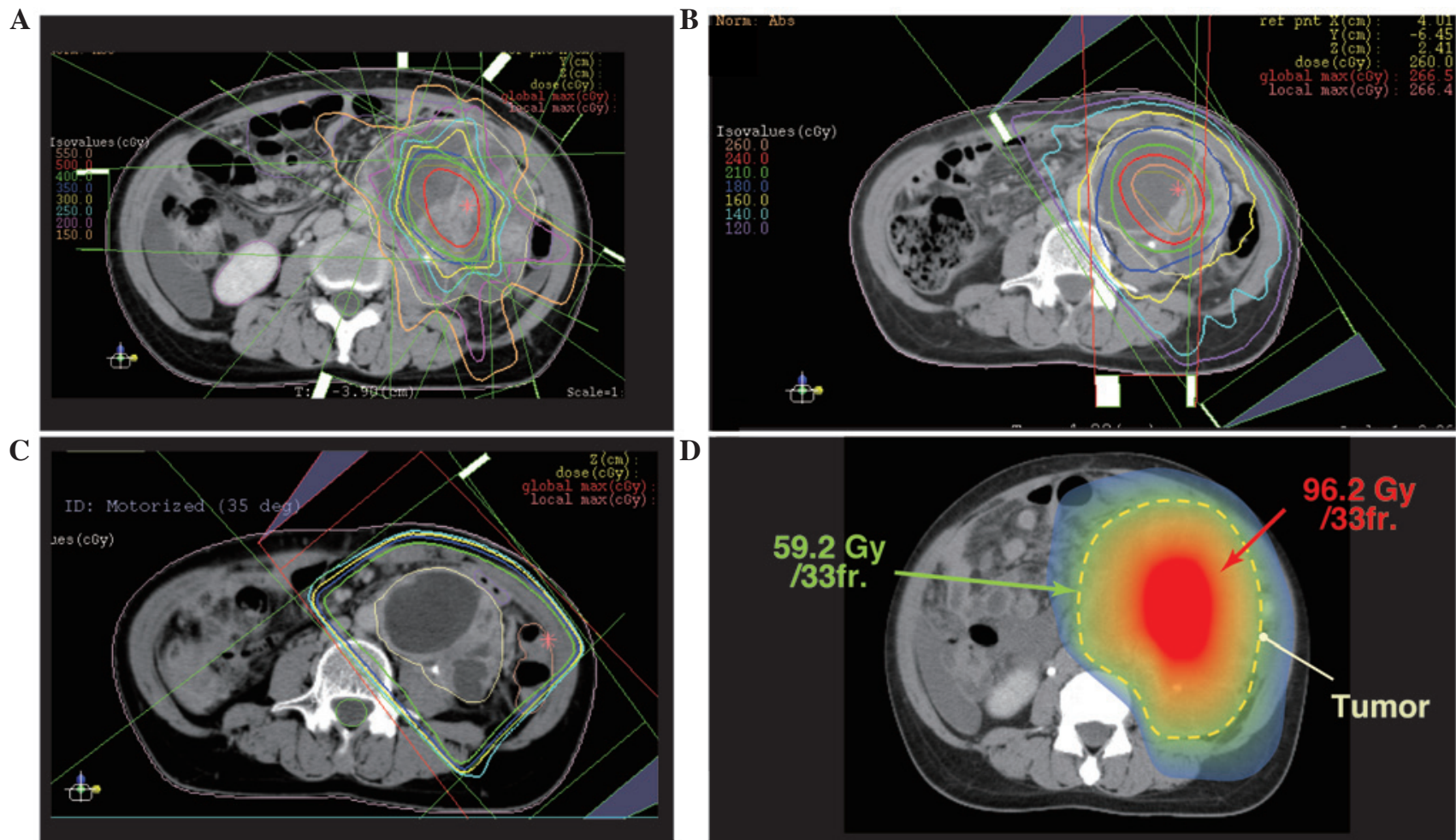

Figure 3. (A) The second initial RTP with modified SIB technique. Field size was adjusted for tumor shrinkage. (B) The third RTP with modified SIB technique, consisting of one conformal arc and two static fields. (C) The fourth RTP with standard technique of three static fields. (D) Cumulative dose distribution of all RTPs (created image). The center part of the tumor was irradiated with >100 Gy in EQD2Gy, whereas the dose to the surrounding tissue was suppressed to <60 Gy in EQD2Gy. RTP, radiotherapy planning; SIB, simultaneous integrated boost; EQD2Gy, equivalent dose in 2 Gy/fraction.

and the patient was subsequently referred to the Department of Urology (Yamagata University Hospital).

A CT-guided core-needle biopsy of the retroperitoneal mass was performed. The mass, which was $>10 \mathrm{~cm}$ in maximum diameter, and was determined to be a poorly-differentiated adenocarcinoma. An immunohistochemical examination revealed the following characteristics of the tumor: Cytokeratin (CK)7(+), CK20(-), CK5/6(-), carcinoembryonic antigen (CEA) (-), and carletinin(-). A CT scan showed a large tumor of $12 \times 16 \times 16 \mathrm{~cm}$, with heterogeneous enhancement and irregular borders, located in the left retroperitoneum (Fig. 1). Left ureteral involvement of the tumor (Fig. 1A and C, black arrowheads) and secondary hydronephrosis were detected, however, there was no continuity between the tumor and the pancreas, adrenal glands, kidneys or stomach. The tumor was determined to be a poorly-differentiated adenocarcinoma arising from the left ureter (12). Although a small amount of ascites was observed in the abdomen, no definite peritoneal dissemination or distant metastasis was identified. The patient's plasma levels of carbohydrate antigen (CA)-125, CA19-9, and CEA were 201.3 U/ml (normal, <40 U/ml), $16.5 \mathrm{U} / \mathrm{ml}$ (normal, <37 U/ml), and $1.42 \mathrm{ng} / \mathrm{ml}$ (normal, $<5 \mathrm{ng} / \mathrm{ml}$ ), respectively; of these, only CA-125 levels were abnormally elevated.

Definitive surgery was initially scheduled. However, due to the infiltration and rapid growth of the tumor, and the poor general condition of the patient, the possibility of surgery was excluded. The patient was referred to the Department of Radiation Oncology for palliation. She was unable to consume meals due to the large size of abdominal tumor, and her

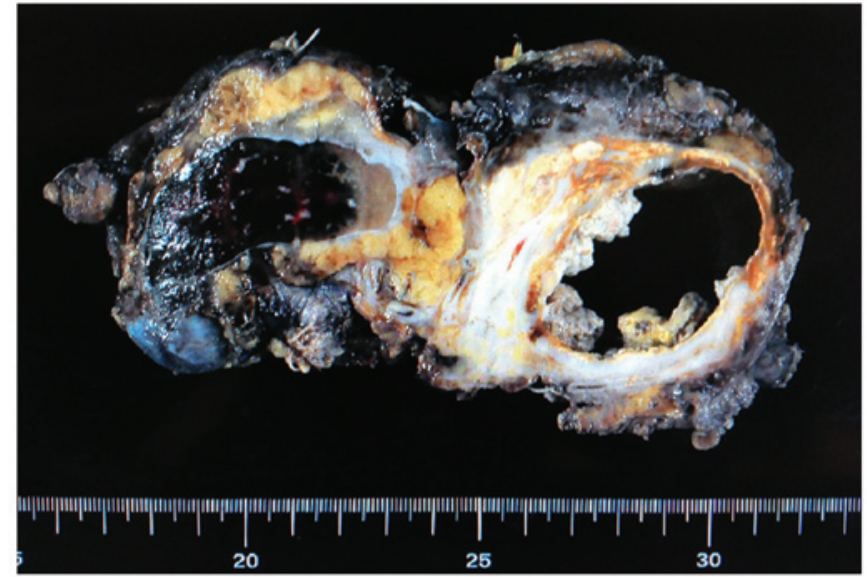

Figure 4. Macroscopic findings of the resected tumor. Cystic components included necrotic tissue, and the surrounding tissue was characterized by xanthogranulomatous changes or fibrosis. The left ureter was unclear due to degeneration and scarring. There was no malignancy.

Eastern Cooperative Oncology Group performance status was determined to be 3 (13).

The initial radiation treatment consisted of coplanar, eight static field three-dimensional-conformal radiotherapy. The treatment was planned to irradiate the center of the tumor with 5 Gy/fraction, and the border area of the tumor and healthy tissue with $<2$ Gy/fraction (Fig. 2). In total, the center of the tumor was irradiated with $25 \mathrm{~Gy} / 5$ fractions and the border area of the tumor and healthy tissue was irradiated with 
$10 \mathrm{~Gy} / 5$ fractions. Subsequently, the center of the tumor was irradiated with an additional $20 \mathrm{~Gy} / 4$ fractions (Fig. 3A) and 26 Gy/10 fractions (Fig. 3B) using a modified simultaneous integrated boost (SIB) technique, and with $25.2 \mathrm{~Gy} / 14 \mathrm{frac}-$ tions using a standard technique (Fig. 3C). The cumulative dose to the center of the tumor and border area of the tumor was $96.2 \mathrm{~Gy} / 33$ fractions and $59.2 \mathrm{~Gy} / 33$ fractions, respectively (Fig. 3D). The equivalent dose in 2 Gy/fraction (EQD2Gy) was calculated using the following equation: EQD2Gy $=$ (total dose $) \times($ dose/fraction $+\alpha / \beta) /(2+\alpha / \beta)(14)$, and the $\alpha / \beta$ of the tumor was assumed to be 10 (15). The EQD2Gy of the center of the tumor was calculated to be $108 \mathrm{~Gy}$, and the EQD2Gy of the surrounding healthy tissue was calculated to be $\sim 58$ Gy. This radiotherapy with modified SIB technique was approved as a clinical trial by the Institutional Review Board of Yamagata University Hospital.

Almost no side effects were caused by radiation therapy during the course of irradiation. Symptoms caused by the tumor decreased with the progression of the treatment, and the performance status at the completion of irradiation treatment was 0 . The size of the tumor at the end of the irradiation was $7 \times 6 \times 15 \mathrm{~cm}$ ( $80 \%$ reduction in volume), and the cystic components and fibrotic septum without enhancing solid components were dominant in the tumor (Fig. 3C). After 47 days from the final day of irradiation, the patient underwent a combined resection of the tumor and adjacent organs (including part of the peritoneum and small bowel). Although no tumor regrowth was observed, the resection was performed for confirmation of tumor response as the patient's physical condition had improved and was considered suitable for surgery.

The resected tumor is shown in Fig. 4. Almost all of the cystic component was necrotic tissue, and xanthogranulomatous changes and fibrosis were observed in the peripheral region. No residual adenocarcinoma cells were observed (pathologically complete response). The ureter that was located in the center of the tumor was replaced by fibrotic tissue due to tumor invasion and irradiation, and pyelonephritis and hydronephrosis were observed in the left kidney. The patient has been recurrence-free for 3 years and 3 months, with no metastasis or complications.

\section{Discussion}

Soft tissue sarcoma or lymphoproliferative disorders are dominant in retroperitoneal neoplasms $(1,3,4)$. Various types of adenocarcinoma of the retroperitoneum have been reported, including pancreatic cancer, renal cell carcinoma, mucinous adenocarcinoma (16,17), Müllerian adenocarcinoma (18), cystadenocarcinoma (19) and adenocarcinoma arising from retroperitoneal teratoma (20).

Surgical resection is typically used for the treatment of retroperitoneal malignant tumors, including soft tissue sarcoma, pancreatic cancer, adrenal cancer (3), whilst chemotherapy with/without radiotherapy is used for the treatment of lymphoproliferative disorders $(21,22)$. Non-hematological tumors, including sarcoma, adenocarcinoma and neurogenic tumors, are usually insensitive to radiation. Furthermore, although microscopic lesions may be controlled with intraoperative or postoperative radiotherapy $(23,24)$, radiotherapy is not used as the definitive treatment of retroperitoneal tumors. The tolerance dose of abdominal organs, such as the small intestine and duodenum, is $\leq 60$ Gy in EQD2Gy; however, adenocarcinomas of $>10 \mathrm{~cm}$ cannot be controlled with a total dose of $60 \mathrm{~Gy}$, thus the required dose for control of large retroperitoneal tumors exceeds the tolerance dose of healthy tissues.

In the present case, modified SIB radiotherapy was used. The standard SIB technique can create contrasts in the dose distribution, and is used for head and neck or brain tumors $(25,26)$. However, the dose distribution in the tumor is homogeneous in the standard SIB technique, and the dose received by the tumor usually does not exceed the tolerance dose of the surrounding healthy tissue. With the modified SIB technique, it is possible to irradiate the center of tumor with higher doses than the surrounding healthy tissue, by creating contrasts in the dose distribution in the tumor $(27,28)$. This difference between the standard SIB technique and modified SIB technique is demonstrated in the present study. For example, as a single dose of $5 \mathrm{~Gy}$ is dangerous for the digestive tract, it is typically not used in abdominal radiotherapy. However, in the current patient, the modified SIB technique was used to safely irradiate the tumor with $5 \mathrm{~Gy} /$ fraction by reducing the dose to the surrounding tissue to $<2 \mathrm{~Gy} /$ fraction (Fig. 2). The total irradiation dose received by the center of the tumor was calculated to be $108 \mathrm{~Gy}$ in EQD2Gy. Although this dose is not used in standard radiotherapy, the modified SIB technique allowed the safe administration of the scheduled radiotherapy. In addition, surgical resection was successfully performed without complications.

In conclusion, recent advanced technologies in radiotherapy have enabled the planning of precise and complex radiotherapy in the present case. The modified SIB technique is a new method that is considered to change the conventional concept that the irradiation dose must not exceed the tolerance dose of healthy tissues. This technique is particularly effective for large tumors in which a dose higher than the tolerance dose of healthy tissues is necessary. Furthermore, as demonstrated by the present case, complete recovery can be achieved for patients who cannot undergo surgery due to the advanced nature of the tumor. Modified SIB radiotherapy may be used in abdominal tumors and also in tumors arising at other sites (27). Future challenges include the establishment of a technique for the use of modified SIB radiotherapy and increasing the number of enrolled patients.

\section{References}

1. Van Roggen JF and Hogendoorn PC: Soft tissue tumours of the retroperitoneum. Sarcoma 4: 17-26, 2000.

2. Strauss DC, Hayes AJ and Thomas JM: Retroperitoneal tumours: Review of management. Ann R Coll Surg Engl 93: 275-280, 2011.

3. Strauss DC, Hayes AJ, Thway K, Moskovic EC, Fisher C and Thomas JM: Surgical management of primary retroperitoneal sarcoma. Br J Surg 97: 698-706, 2010.

4. Clark MA, Fisher C, Judson I and Thomas JM: Soft-tissue sarcomas in adults. N Engl J Med 353: 701-711, 2005.

5. Menenakos C, Braumann C, Hartmann J and Jacobi CA: Retroperitoneal Castleman's tumor and paraneoplastic pemphigus: report of a case and review of the literature. World J Surg Oncol 5: 45, 2007.

6. Zerrweck-López C, Quijano-Orvañanos F, Montañez-Ramírez H, Murillo-Zolezzi A, Toledo-Valdovinos S and Padilla-Longoria R: Neuroblastoma in the adult. Case report. Cir Cir 77: 397-401, 2009.

7. Kattepura S, Das K, Correa MM and Devarabhavi H: Giant gastrinoma in a child: Case report and review. Pediatr Surg Int 24: 1083-1085, 2008. 
8. Tasdemir A, Soyuer I, Yurci A, Karahanli I and Akyildiz H A huge solitary fibrous tumor localized in the pancreas: A young women. JOP 13: 304-307, 2012.

9. Gatcombe HG, Assikis V, Kooby D and Johnstone PA: Primary retroperitoneal teratomas: A review of the literature. J Surg Oncol 86: 107-113, 2004.

10. Li Z, Xiang J, Yan S, Gao F and Zheng S: Malignant triton tumor of the retroperitoneum: A case report and review of the literature. World J Surg Oncol 10: 96, 2012.

11. Buse S, Gilfrich C, Wagener N, Pfitzenmaier J, Haferkamp A and Hohenfellner M: Thoraco-abdominal approach to large retroperitoneal tumours. BJU Int 98: 969-972, 2006.

12. National Comprehensive Cancer Network: Occult Primary, Version 3.2014. http://www.nccn.org/professionals/physician gls/pdf/occult.pdf. Accessed July 9, 2014.

13. Oken MM, Creech RH, Tormey DC, Horton J, Davis TE, McFadden ET and Carbone PP: Toxicity and response criteria of the Eastern Cooperative Oncology Group. Am J Clin Oncol 5: 649-655, 1982.

14. Joiner MC: A simple alpha/beta-independent method to derive fully isoeffective schedules following changes in dose per fraction. Int J Radiat Oncol Biol Phys 58: 871-875, 2004.

15. Stuschke M, Budach V, Budach W, Feldmann HJ and Sack H: Radioresponsiveness, sublethal damage repair and stem cell rate in spheroids from three human tumor lines: Comparison with xenograft data. Int $\mathbf{J}$ Radiat Oncol Biol Phys 24: $119-126,1992$

16. Jiang H, Jin K, You Q, Fang W and Xu N: Retroperitoneal primary mucinous adenocarcinoma: A case report. Oncol Lett 2: 633-636, 2011.

17. Cupp JS, Illeck J, Rahbar N, Rettenmaier MA and Goldstein BH A rare case of primary retroperitoneal mucinous adenocarcinoma: A case report. J Reprod Med 58: 85-88, 2013.

18. Spinelli C, Strambi S, Tartaglia D, Di Franco G, Pucci V, Faviana $\mathrm{P}$ and Lencioni M: Primary retroperitoneal müllerian adenocarcinoma: A case report and literature review. Case Rep Oncol 6: 616-621, 2013.
19. Yadav R, Kataria K, Balasundaram P and Karak AK: Mucinous cystadenocarcinoma arising in an ectopic kidney simulating a retroperitoneal dermoid cyst: A rare tumour presenting as a diagnostic dilemma. Malays J Pathol 35: 95-98, 2013.

20. Ghosal SR,Das S, Maji A,Dey KK and Bagchi D: Adenocarcinoma in retroperitoneal teratoma. Indian J Surg 75: 33-35, 2013.

21. Tambo M, Fujimoto K, Miyake M, et al: Clinicopathological review of 46 primary retroperitoneal tumors. Int J Urol 14 : 785-788, 2007.

22. National Comprehensive Cancer Network: Non-Hodgkin's Lymphomas, Version 2.2014. http://www.nccn.org/professionals/physician_gls/pdf/nhl.pdf. Accessed May 29, 2014.

23. Clark JA and Tepper JE: Role of radiation therapy in retroperitoneal sarcomas. Oncology (Williston Park) 10: 1867-1872, 1996.

24. Thomas DM, O'Sullivan B and Gronchi A: Current concepts and future perspectives in retroperitoneal soft-tissue sarcoma management. Expert Rev Anticancer Ther 9: 1145-1157, 2009.

25. Peponi E, Glanzmann C, Kunz G, Renner C, Tomuschat K and Studer G: Simultaneous integrated boost intensity-modulated radiotherapy (SIB IMRT) in nasopharyngeal cancer. Strahlenther Onkol 186: 135-142, 2010

26. Cho KH, Kim JY, Lee SH, et al: Simultaneous integrated boost intensity-modulated radiotherapy in patients with high-grade gliomas. Int J Radiat Oncol Biol Phys 78: 390-397, 2010.

27. Nomiya T, Akamatsu H, Harada M, et al: Modified simultaneous integrated boost radiotherapy for an unresectable huge refractory pelvic tumor diagnosed as a rectal adenocarcinoma. World J Gastroenterol 20: 18480-18486, 2014.

28. Nomiya T, Akamatsu H, Harada M, Ota I, Hagiwara Y, Ichikawa M, Miwa M, Suzuki A and Nemoto K: Modified simultaneous integrated boost radiotherapy for unresectable locally advanced breast cancer: Preliminary results of a prospective clinical trial. Clin Breast Cancer: Nov 15, 2014 (Epub ahead of print). 